\title{
Análisis del Ciclo de Vida de una Unidad Prototipo de Vivienda de Emergencia. La búsqueda del impacto nulo
}

\section{Life-Cycle Assessment of Prototype Unit of Emergency Housing. The search for the zero impact}

\author{
$\underline{\text { J. M. Ros García }}^{(*)}$, G. Sanglier Contreras ${ }^{(* *)}$
}

RESUMEN

La Unidad Prototipo de Vivienda de Emergencia (UPVE) es el resultado del Proyecto de Investigación Aplicada VEM (Vivienda de Emergencia Militar) desarrollado con la colaboración de la Escuela Politécnica Superior de la Universidad CEU y la empresa Airbus Defense \& Space. Es concebido como una unidad modular e industrializable de habitabilidad básica, de crecimiento programado y extensible, diseñada para proporcionar refugio y protección en entornos de crisis humanitaria o en contingencias de vulnerabilidad social con el fin de asegurar un hábitat sostenible de emergencia.La influencia que los procesos de construcción y materiales involucrados en la fabricación de dicha UPVE tienen en el medio ambiente, analizados mediante la metodología de Análisis de Ciclo de Vida (ACV), consideran especialmente crítico el reciclado de los materiales utilizados. Así, con el objetivo de analizar y disminuir el impacto medioambiental, se cuantifican cada una de las partes que componen la Unidad Prototipo desarrollada, valorando los beneficios resultantes de los procesos de fabricación en la disposición final de los materiales según la metodología DfMA (Design for Manufacturing and Assembly).

Palabras clave: ACV; Vivienda de Emergencia Militar; construcción sostenible; DfMA.

\section{ABSTRACT}

Prototype Unit of Emergency Housing (PUEH) is the result of the Applied Research Project VEM (Military Emergency Housing) developed in collaboration with Escuela Politécnica Superior (Universidad CEU) and the company Air-bus Defense \& Space. It is designed as a modular and industrialized unit of basic habitability, with programmed and expandable growth, designed to provide shelter and protection in environments of humanitarian crises or contingencies of social vulnerability in order to ensure sustainable habitat for emergencies. The influence of the construction processes and materials involved in the manufacture of this PUEH have on the environment, analyzed using the methodology of life-cycle assessment (LCA), considered especially critical recycling the mate-rials used. Thus, in order to reduce the environmental impact environmental, each of the component parts of the developed prototype unit are quantified, evaluating the benefits resulting from the methodology DfMA (Design for Manufacturing and Assembly).

Keywords: LCA; Housing of Military Emergency; sustainable construction; DfMA.

\footnotetext{
(*) Profesor Dr. Arquitecto. Investigador Principal Grupo Rebirth-Inhabit. Departamento de Arquitectura y Diseño, Escuela Politécnica Superior, Universidad CEU San Pablo, Madrid (España).

(**) Profesor Dr. Ciencias Físicas, Ingeniero Técnico Aeronáutico. Área de Ingeniería de la Construcción, Escuela Politécnica Superior, Universidad CEU San Pablo, Madrid (España).

Persona de contacto/Corresponding author:jmros.eps@ceu.es (J. M. Ros García)

ORCID: http://orcid.org/oooo-0oo1-8994-3141 (J. M. Ros García);

http://orcid.org/oooo-0oo2-8981-5622 (G. Sanglier Contreras)
}

Cómo citar este artículo/Citation: Ros García, J. M., Sanglier Contreras, G. (2017). Análisis del Ciclo de Vida de una Unidad Prototipo de Vivienda de Emergencia. La búsqueda del impacto nulo. Informes de la Construcción, 69(547): e211, doi: http://dx.doi.org/10.3989/ ic.16.035.

Copyright: (C) 2017 CSIC. Licencia / License: Salvo indicación contraria, todos los contenidos de la edición electrónica de Informes de la Construcción se distribuyen bajo una licencia de uso y distribución Creative Commons Attribution License (CC BY) Spain 3.o. 


\section{INTRODUCCIÓN}

La mayor parte de prototipos ejemplares del pasado de viviendas de emergencia, como la Cooper House (Forster \& Robert, 1933), los Dymaxion Deployment Units (DDUs) (Fuller, 1940), las conocidas Quonset Hut (desarrolladas entre 1941 y el final de la guerra del Pacífico), o la Airform House (Neff, 1946), fueron concebidos para necesidades logísticas militares o situaciones de repoblación civil posbélica. Avanzaron en paralelo con la moderna industrialización apoyados por potentes políticas de subvención pública presentando una progresiva mejora en su evolución, sin embargo, los modelos nunca fueron optimizados para satisfacer elevadas prestaciones de transporte y montaje, exigibles hoy a cualquier unidad de alojamiento extremo, y derivaron a productos de segundo rango, destinados a viviendas de emergencia de menor calidad y con una vida útil corta debido a la rápida obsolescencia de sus materiales de construcción.

Uno de los ejemplos más destacados y visionarios lo lleva a cabo Jean Prouvé en 1949. A partir de su primer modelo de los años 30, la Maison Standard Metropole, desarrolla un verdadero manifiesto arquitectónico con sus tres prototipos de la Maison Tropicale, demostrando cómo su construcción prefabricada y modulada de acero y aluminio es capaz de integrarse perfectamente a un medio natural concreto, incluso mejorando los modelos de la tradición local, y aportando como grandes ventajas la optimización del embalaje, transporte y montaje industrializado.

En la situación actual y sobre todo a partir de la última década del siglo XX, en que se asentaron las bases del movimiento conocido como NUEVO HUMANITARISMO, los modelos habituales y más extendidos de agrupación rápida de las conocidas tipológicamente como "Viviendas de Emergencia», unidades básicas de refugio convencionales (tipo tienda con telas o contenedores metálicos), generan a corto plazo un problema añadido de insalubridad, de hacinamiento y de falta de adaptación al medio natural.

El presente estudio se desprende de los resultados del Proyecto de Investigación VEM (1) (2) en el que participaron la Universidad CEU-San Pablo (Grupo de Investigación REBIRTHINHABIT del Departamento de Arquitectura y Diseño) y la empresa AIRBUS Defense \& Space, del que se obtuvo la concesión de una patente en explotación («Arquitectura de alojamiento adaptable modular» (sistema 3a) «Modular adaptable housing architecture» P201330097 - WIPO/PCT : WO 2014/114836 A1) para el modelo de Vivienda de Emergencia (VE) que se ha analizado en este trabajo desde el punto de vista del impacto medioambiental, mediante un Análisis de Ciclo de Vida, considerando los efectos del reciclado de los materiales utilizados en la fabricación de la Unidad Prototipo llevada a cabo para su validación. Utilizando además la metodología concurrente DfMA se pretende recortar los costes de producción derivados de la fabricabilidad de componentes eficientes del sistema constructivo analizado, considerando los distintos requerimientos del ACV e implicándolo en las decisiones del diseño.

\section{OBJETIVO Y ALCANCE (3)}

Los estudios de Análisis de Ciclo de Vida sobre viviendas de emergencia de este tipo son prácticamente inexistentes en la literatura actual, por ello se considera de gran interés este trabajo en cuanto a la aplicación de esta metodología a las características de estas viviendas. El ACV identifica y cuantifica el uso de materiales, energía y emisiones al medio ambiente para evaluar el impacto medioambiental que producen estas cargas. A partir de estos resultados permite proponer estrategias de mejora ambiental. El presente estudio contempla el análisis y la evaluación del ciclo de vida de una Unidad Prototipo de Vivienda de Emergencia con el objetivo de identificar los procesos o sustancias más nocivas para el medio ambiente con el fin de aplicar las medidas correctoras necesarias. Una vez constatada la escasez de estudios existentes de ACV sobre estos modelos de vivienda, con este análisis se pretende ofrecer datos de referencia registrados experimentalmente del prototipo construido, aportando su caso particular. Así mismo, su objetivo principal ha sido establecer una relación ponderada entre los diferentes materiales del sistema constructivo propuesto, buscando el impacto mínimo y aportando soluciones de compromiso.

En el presente estudio se define como unidad funcional a analizar la cantidad de material y energía necesaria para construir una Unidad Prototipo de la Vivienda de Emergencia, estudiando los impactos ambientales producidos en el medio ambiente, atendiendo a tres categorías genéricas de impacto (endpoints) y analizando los diferentes indicadores que componen cada una de ellas. También se ha estudiado el impacto de los mismos materiales introduciendo técnicas de reciclado y gestión final del producto. Instituciones como el Programa de Medio Ambiente de las Naciones Unidas (mediante la ENEP-SETAP Life Cycle Initiative) o la Comisión Europea (mediante la Plataforma Europea de ACV) promueven la aplicación de estudios de este tipo para la mejora ambiental y procesos en todo tipo de sectores, incluyendo la construcción. Este análisis se ha realizado de acuerdo a las normas internacionales ISO:14040 (4), ISO:14041 (5), ISO:14042 (6), ISO:14043 (7) e ISO:14044 (8). Sin embargo, existen otras normas nacionales importantes para la realización de este tipo de análisis, como la UNE-EN 15804 (9), UNE-EN 15978:2012 (10) y UNE-EN 15643 (11) que están sometidas a normas ISO y CEN (Norma ISO:21930) (12).

En la norma española (13) se establece que «el ACV es una recopilación y evaluación, conforme a un conjunto sistemático de procedimientos, de las entradas y salidas de materia y energía, y de los impactos ambientales potenciales directamente atribuibles a la función del sistema del producto a lo largo de su ciclo de vida».

El perfil de datos (14) seleccionado para este trabajo ha sido el siguiente:

- Periodo: 2010 y posterior

- Geografía: datos mixtos.

- Tecnología: datos mixtos.

- Representatividad: promedio de todos los proyectos.

- Sustitución de asignación: no aplicable.

- Asignación para salidas múltiples: no aplicables.

- Reglas de corte: menos del 5 \% (criterios físicos).

- Límite con la naturaleza: desconocido.

- Infraestructura: no.

- Base de datos a emplear para datos de fondo: Ecoinvent v3.o.

- Método de recolección de datos de primer plano: datos aportados por las empresas de fabricación de las diferentes partes de la Unidad Prototipo. 


\subsection{Limitaciones}

Como recomiendan varios consultores de ACV, no se han tenido en cuenta las entradas y salidas que tengan menos del 5 $\%$ de la masa total del inventario, salvo aquellas que representen componentes tóxicos o peligrosos. Los efectos regionales y temporales no se han incluido por las insuficiencias en la metodología (ReCiPe) y en la base de datos (Ecoinvent) utilizados en este trabajo.

En el ciclo de vida de cualquier producto actúan múltiples sistemas dependientes unos de otros y que deberían de tenerse en cuenta (todo proceso está vinculado a otro subproceso, y así sucesivamente). El análisis de todos estos procesos sería interminable por su escala, por ello se hace necesario establecer sus límites a modo de simplificaciones o hipótesis en las diferentes fases del inventario del ACV (15). Este estudio de un prototipo de VEM se establece como un ACV simplificado de segundo orden.

Debido a los escasos estudios existentes de ACV sobre estos modelos de vivienda, no es posible actualmente realizar un análisis comparativo entre el modelo de vivienda de emergencia propuesto y otros similares. En este sentido, el presente estudio se limita al análisis del impacto ambiental del modelo propuesto, con la intención de definir un antecedente a nuevas aportaciones que puedan producirse en dicho campo.

Genéricamente, en la esquematización de un ciclo de vida se han de considerar los siguientes pasos:

- La adquisición de materia prima.

- La manufactura, el procesado y la formulación.

- La distribución y el transporte.

- El uso y la reutilización y mantenimiento.

- Reciclado.

- Gestión de residuos.

\section{APLICACIÓN A LA UNIDAD PROTOTIPO}

Como se ha explicado anteriormente, se analiza y evalúa el ciclo de vida de una Unidad Prototipo de Vivienda de Emergencia. Abarca el análisis desde la obtención de la materia prima hasta su uso final cradle to gate o, si se respetan los esfuerzos de reciclaje/reutilización, como es el caso, cradle to cradle, no completo en este estudio debido a la falta de información en la fase uso/mantenimiento. Se tienen en cuenta los flujos principales de recursos en las diferentes etapas del ciclo de vida.

Esta Vivienda de Emergencia está pensada para proporcionar refugio y protección a una familia de cinco miembros en situaciones de emergencia humanitaria (catástrofes naturales, conflictos bélicos, etc.) y se compone de dos unidades modulares del «kit básico de emergencia» (estructura + envolventes + suelo + faldón) con una vida útil máxima de tres años. Dentro de los objetivos se ha pretendido que dicha vivienda fuera capaz de ser montada de forma rápida y sencilla, incluso por personal poco formado, y ampliada y modificada de forma progresiva según las necesidades del momento. En este estudio de ACV se ha analizado una sola Unidad Prototipo, ya que las dos unidades que conforman la vivienda son idénticas.

La optimización de la geometría de este tipo de vivienda adquirió gran relevancia en la fase de diseño por su implicación en diversos factores de su construcción y vida útil. La relación entre la superficie envolvente y el volumen contenido por la misma resulta un aspecto fundamental que influye en la utilización de recursos para su construcción, el dimensionado estructural, las dimensiones y pesos involucrados en su embalaje, transporte y montaje y el balance energético de la vivienda durante su funcionamiento. Esta relación recibe la denominación de factor de forma y el objetivo de su optimización en este proyecto se basó en conseguir el mayor volumen interior posible que fuera habitable contenido dentro de la menor superficie de envolvente funcional, constituyendo ésta una gran parte de los elementos constructivos de la vivienda.

El factor de forma resultó uno de los aspectos fundamentales en la consideración de las diferentes opciones geométricas que fueron analizadas, presentando la propuesta definitiva una relación de superficie de envolvente y volumen contenido de 1,21. Se estimó que, en este orden de dimensiones, el rango eficiente del factor de forma se encuentra entre 1 y 1,5, siendo 1 el valor óptimo (relacionado con la geometría de la esfera). El valor de la propuesta definitiva es de 1,21, sensiblemente inferior al de otras opciones estudiadas en el proyecto, como se muestra en la Figura 1.

De la optimización del factor de forma en este proyecto se deriva el ahorro de materiales utilizados en la construcción de la envolvente (entre un 3 y $19 \%$ menos superficie que otros modelos de vivienda de emergencia) y su estructura. Con ello se disminuye del impacto ambiental que conlleva, además de facilitar el embalaje, transporte y montaje de estas viviendas. Otra consecuencia del factor de forma a tener en cuenta en relación al impacto ambiental es su influencia en el balance energético durante el uso de la vivienda. La reducción de esta relación geométrica implica la disminución de la superficie de intercambio energético con el exterior respecto al volumen interior habitado, que actúa como acumulador de energía.

La Unidad Prototipo resultante, y analizada en este estudio, es de base cuadrada y tiene unas dimensiones (largo $\times$ ancho $\times$ alto útil) de $3 \times 3 \times 2,5 \mathrm{~m}$; la Vivienda de Emergencia estará compuesta por dos unidades prototipos. El peso total de una Unidad Prototipo es de aproximadamente unos $227 \mathrm{~kg}$ (454 kg para la Vivienda de Emergencia). La Unidad Prototipo está compuesta principalmente de seis partes (Figura 2):

- Estructura principal formada por:

- un soporte inferior de acero al carbono S-275-JR según la norma EN 10025 (16);

- un soporte o cubierta superior de aleación de aluminiomagnesio-silicio $6005 \mathrm{~A}$ con tratamiento térmico T4.

- Membranas o envolventes:

- la envolvente exterior de tejido plano $100 \%$ poliéster de ligamento tafetán con recubrimiento de PVC por ambas caras con una masa/unidad de superficie de $500 \mathrm{~g} / \mathrm{m}^{2}$;

- la envolvente interior de poliéster $100 \%$ de alta tenacidad recubierto de algodón con una masa/unidad de superficie de $135 \mathrm{~g} / \mathrm{m}^{2}$.

- Base/Suelo: panel sándwich de núcleo de poliuretano recubierto de dos planchas de acero inoxidable $\mathrm{X}_{5} \mathrm{CrNi}$ 18-10 (1-4301) (según normativa EN 10088-1:2015) (17).

- Faldón perimetral: compuesto de un geotextil de polipropileno y poliéster. 


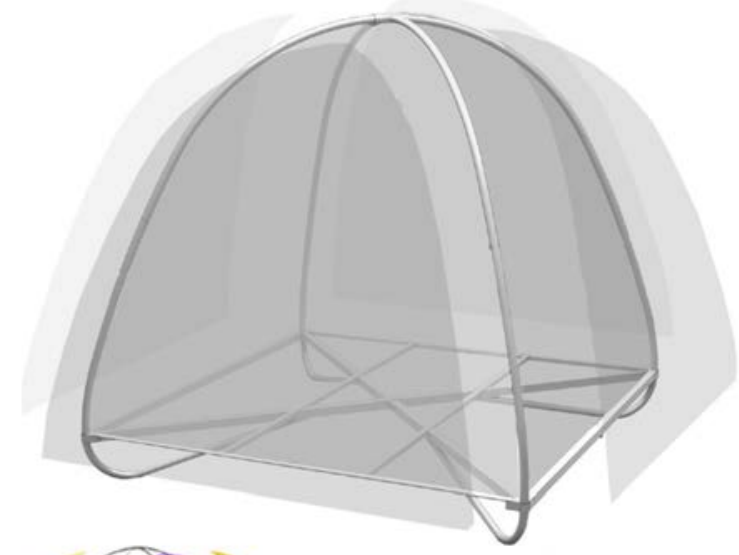

\section{Ejemplos más extendidos}

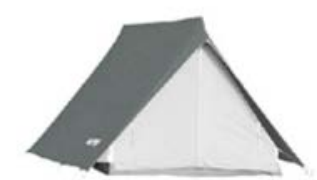

$F f=1.25$

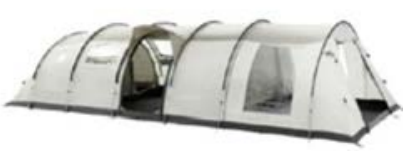

$\mathrm{Ff}=\mathbf{1 . 3 2}$

\section{Proyecto VEM}

Eficiencia en el factor de forma:

$$
\mathrm{Ff}=\mathrm{Se} / \mathrm{Ve}=\mathbf{1 . 2 1}
$$

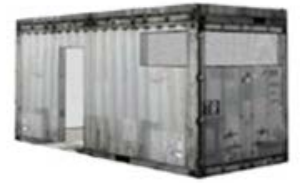

(Se) : $43.61 \mathrm{~m}^{2}$

Volumen interior

(Ve) : $35.97 \mathrm{~m}^{3}$

\section{EI rango eficiente se estima entre 1-1.5}

$F f=1.5$

Figura 1. Cálculo y comparativa del «factor de forma».

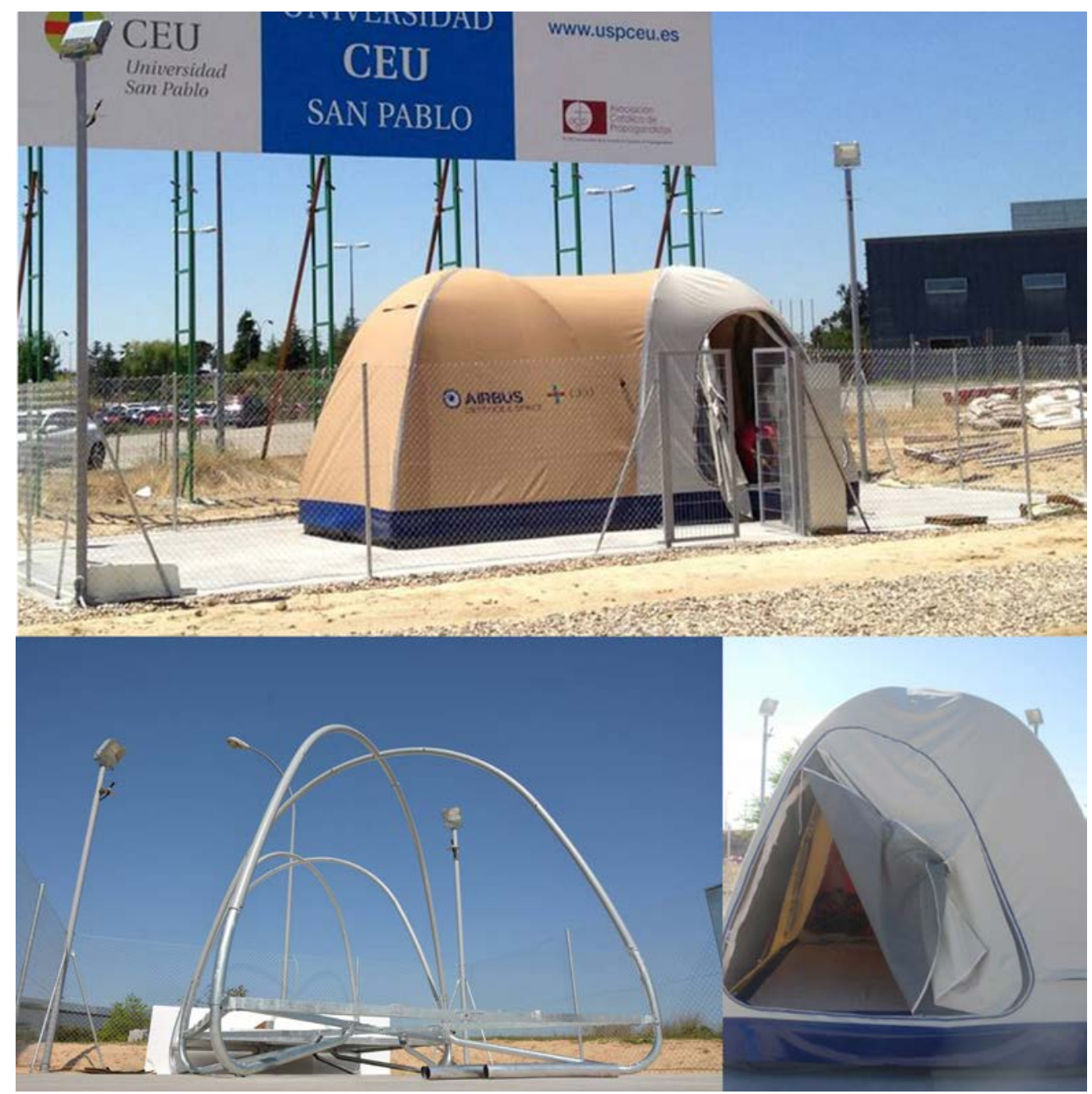

Figura 2. Vivienda de Emergencia Militar compuesta por dos Unidades Prototipo, estructura metálica, envolventes exterior e interior y faldón perimetral de la Unidad Prototipo desarrollada. 
A continuación se presenta un diagrama de flujo simplificado (Figura 3) considerándose que este producto es de segundo orden, el necesario establecido para cualquier ACV. Se representa material, equipos, energía, transporte y procesos.

La contribución al peso total de las diferentes partes de la Unidad Prototipo se recoge en la Tabla 1 y las composiciones químicas y masas de los diferentes elementos metálicos (apor- tadas por los fabricantes) en la Tabla 2 (17) (18) (19). Para el acero al carbono se ha utilizado un tratamiento de galvanizado y para las piezas de aluminio se ha aplicado un pre-tratamiento de cromatizado con espesor de película 60-80 micras y un tratamiento posterior de lacado a base de tipo linterpon D1036 de 83 micras de espesor. Los datos para la producción de PET (poliéster) y PP (polipropileno) se han estimado según los datos proporcionados para los otros termoplásticos.

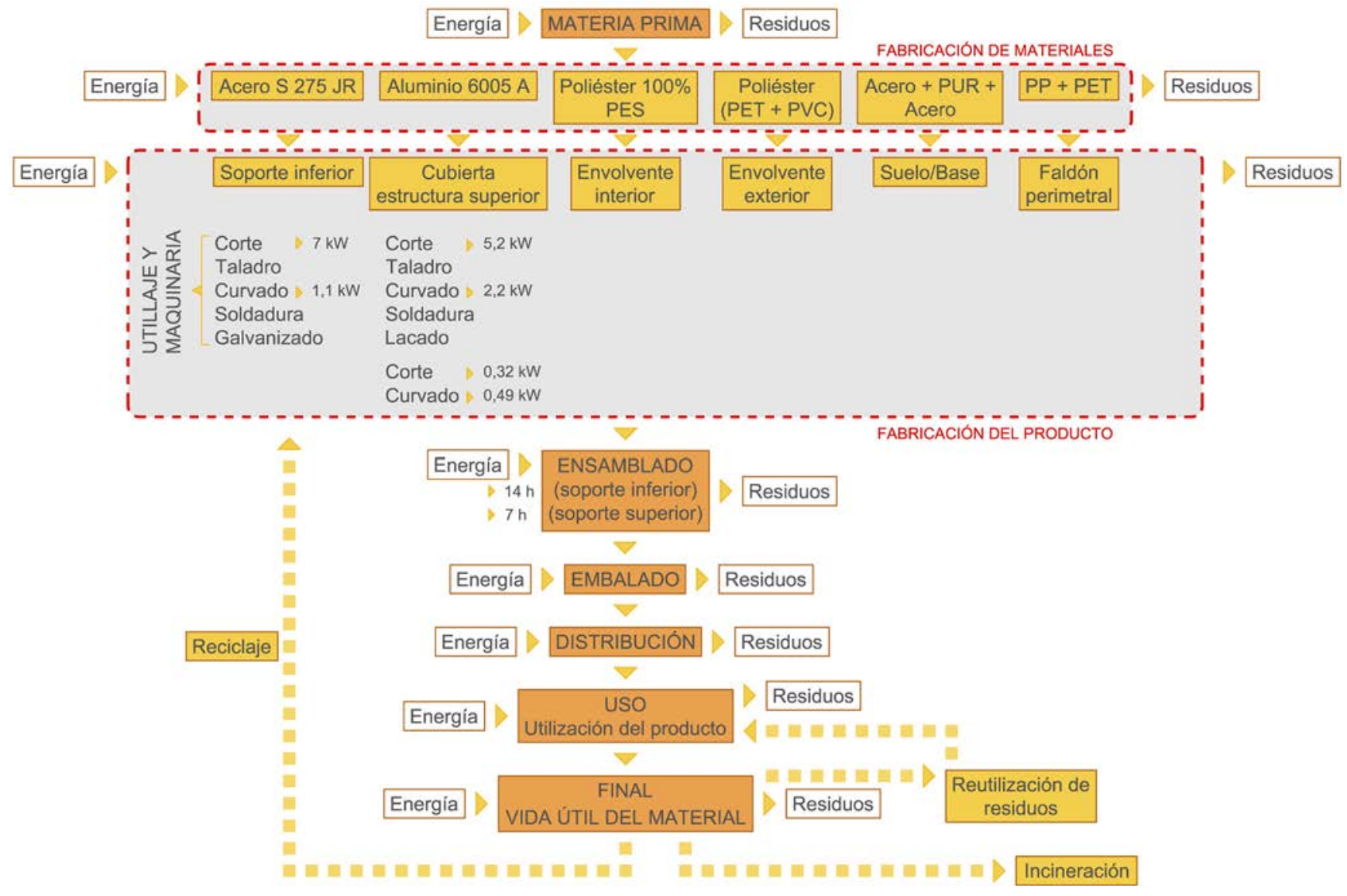

Figura 3. Flujograma de la Unidad Prototipo desarrollada (Fuente: Original propia).

Tabla 1. Peso, energía y contribuciones de cada una de las partes de la Unidad Prototipo.

\begin{tabular}{|c|c|c|c|c|c|c|}
\hline $\begin{array}{c}\text { Partes de la Unidad } \\
\text { Prototipo }\end{array}$ & Material & $\begin{array}{l}\text { Peso } \\
\text { (kg) }\end{array}$ & $\begin{array}{c}\text { Energía } \\
(\mathbf{k W h} / \mathbf{k g})\end{array}$ & $\begin{array}{l}\text { Unidad } \\
\text { (kWh) }\end{array}$ & $\begin{array}{c}\text { Vivienda } \\
\text { (kWh) }\end{array}$ & \\
\hline Soporte principal + accesorios & Acero & 108,60 & 11,94 & $1.296,68$ & $2.593,37$ & Acero \\
\hline Suelo/Base & Acero inox & 57,10 & 11,94 & 681,73 & $1.363,45$ & Acero \\
\hline Cubierta superior & Aluminio & 13,83 & 44,44 & 614,61 & $1.229,21$ & Aluminio \\
\hline Envolvente interior & PET & 4,73 & 24,00 & 113,40 & 226,80 & Plástico \\
\hline Envolvente exterior & PET & 12,60 & 24,00 & 302,40 & 604,80 & Plástico \\
\hline Envolvente exterior & $\mathrm{PVC}$ & 4,90 & 22,22 & 108,88 & 217,76 & Plástico \\
\hline Suelo/Base & PUR & 10,94 & 19,44 & 212,75 & 425,50 & Plástico \\
\hline Suelo/Base & PS & $4,73 \mathrm{E}-\mathrm{O} 4$ & 27,78 & 0,01 & 0,03 & Plástico \\
\hline Suelo/Base & $\mathrm{PVC}$ & 5,00E-03 & 22,22 & 0,11 & 0,22 & Plástico \\
\hline Faldón perimetral & $\mathrm{PP}$ & 3,98 & 20,00 & 79,60 & 159,20 & Plástico \\
\hline Faldón perimetral & PET & 2,62 & 24,00 & 62,88 & 125,76 & Plástico \\
\hline Galvanizado & Zinc & 5,97 & 15,00 & 89,52 & 179,04 & Zinc \\
\hline Cromado & Cromo & 1,41 & 15,00 & 21,14 & 42,27 & Cromo \\
\hline Lacado & PET & 0,34 & 27,78 & 9,36 & 18,72 & Plástico \\
\hline Embalaje plástico retráctil & PEBD o LDPE & 0,13 & 20,83 & 2,73 & 5,46 & Plástico \\
\hline \multirow[t]{2}{*}{ Palet (madera de pino) } & Madera & 50 & 0,83 & 41,5 & 83 & Madera \\
\hline & Total & 277,15 & & $3.637,29$ & $7.274,58$ & \\
\hline
\end{tabular}


Tabla 2. Porcentajes y pesos para los diferentes elementos del acero S275JR, acero inoxidable $\mathrm{X}_{5} \mathrm{CrNi}$ 18-10 y aluminio 6005 A.

\begin{tabular}{|c|c|c|c|c|c|c|c|c|}
\hline $\begin{array}{c}\text { Acero } \\
\text { S275JR } \\
(108,60 \mathrm{~kg})\end{array}$ & $\begin{array}{c}\text { Composición } \\
\text { (\%) }\end{array}$ & $\begin{array}{c}\text { Peso } \\
\text { (kg) }\end{array}$ & $\begin{array}{c}\text { Acero } \\
\text { inoxidable } \\
\text { X5CrNi } \\
18-10 \\
(57,096 \mathrm{~kg})\end{array}$ & $\begin{array}{c}\text { Composición } \\
\text { (\%) }\end{array}$ & $\begin{array}{l}\text { Peso } \\
\text { (kg) }\end{array}$ & $\begin{array}{c}\text { Aluminio } \\
\text { 6005 A (T4) } \\
(13,83 \mathrm{~kg})\end{array}$ & $\begin{array}{c}\text { Composición } \\
\text { (\%) }\end{array}$ & $\begin{array}{c}\text { Peso } \\
\text { (kg) }\end{array}$ \\
\hline $\mathbf{F e}$ & 99,174 & 107,703 & $\mathrm{Fe}$ & 69,760 & 39,830 & Al & 96,800 & 13,387 \\
\hline C & 0,112 & 0,122 & C & 0,070 & 0,040 & $\mathbf{F e}$ & 0,350 & 0,048 \\
\hline Mn & 0,514 & 0,559 & Mn & 2,000 & 1,142 & Mn & 0,500 & 0,069 \\
\hline $\mathbf{P}$ & 0,017 & 0,018 & $\mathbf{P}$ & 0,046 & 0,026 & Mg & 0,550 & 0,076 \\
\hline $\mathbf{S}$ & 0,012 & 0,014 & $\mathbf{S}$ & 0,016 & 0,009 & $\mathrm{Cu}$ & 0,300 & 0,042 \\
\hline Si & 0,146 & 0,158 & $\mathbf{N}$ & 0,110 & 0,063 & Si & 0,900 & 0,125 \\
\hline Al & 0,024 & 0,026 & $\mathbf{C r}$ & 18,000 & 10,277 & $\mathrm{Cr}$ & 0,300 & 0,042 \\
\hline & & & Ni & 10,001 & 5,710 & Zn & 0,200 & 0,028 \\
\hline & & & & & & Ti & 0,100 & 0,014 \\
\hline
\end{tabular}

Conviene resaltar que en la tabla anterior se han considerado los elementos de unión incluidos en la parte de soporte principal + accesorios y que corresponden a las escuadras, bulones, pasadores, tornillos, arandelas y tuercas que representan $13,5 \mathrm{~kg}$ del material de acero total $(108,6 \mathrm{~kg})$ de la Unidad de vivienda y que corresponde a un consumo de energía de $120,96 \mathrm{kWh}$. Esto se ha considerado en la parte de reciclaje y reutilización del presente trabajo, ya que estos materiales pueden ser desmontados sin disminuir sus prestaciones y permiten ser reutilizados.

Se han introducido datos de mano de obra (aportados por las empresas implicadas en los diferentes procesos de elaboración), maquinaria y consumo de potencia de los procesos de fabricación de los diferentes elementos de los que se han dispuesto de datos. Se han considerado también los impactos ambientales debidos a los diferentes desplazamientos del material durante los procesos de fabricación y aplicación de tratamientos hasta los puntos de control de calidad y verificación del producto, así como de la vuelta de estos materiales a su punto de origen y al lugar de montaje último de la Unidad Prototipo.

Se ha tenido en cuenta la fase de embalaje/empaquetado considerando el uso de un palet europeo reciclado y homologado de madera de pino de dimensiones $2.300 \times 1.370 \times 145 \mathrm{~mm}$ con soporte de carga hasta $2.000 \mathrm{~kg}$ y envuelta en políme- ro transparente retráctil tipo olefínico LDPE (polietileno de baja densidad) de 15 micras de espesor. No se ha considerado la energía consumida en la fabricación del palet ni la debida al número de operarios. Cumpliendo con las características propias de la metodología DfMA en Ingeniería Concurrente (IC), todos los componentes constructivos de las partes que integran el sistema han sido diseñados de tal manera que ninguno de ellos excede en su longitud de $2 \mathrm{~m}$, ni peso de 25 $\mathrm{kg}$. Se encuentran paquetizados de acuerdo a sus condiciones de forma y contorno para alcanzar el mayor nivel de adaptación eficiente al volumen de carga estandarizado exigido por AIRBUS Defense \& Space (Figura 4). Al mismo tiempo, el despiece de dichos componentes ha sido normalizado en su fabricabilidad atendiendo a cuestiones de facilidad de montaje, reducción de número de partes y consumo óptimo de material, con una estimación de ahorro económico de un 20 $\%$ sobre series de producción por encima de 100 unidades preparadas para su implantación.

El transporte, el almacenaje y la distribución del producto son daños crecientes sobre el medio ambiente (emisión de contaminantes del transporte, contaminación acústica, etc.) en la fase de distribución. Se ha considerado que las Unidades Prototipos se van a enviar desde Madrid, punto central de distribución, a las capitales de los siguientes países: Brasil, Colombia, India, Polonia y Camboya, puntos estratégicos de la empresa Airbus. Se supone el uso de un avión CASA
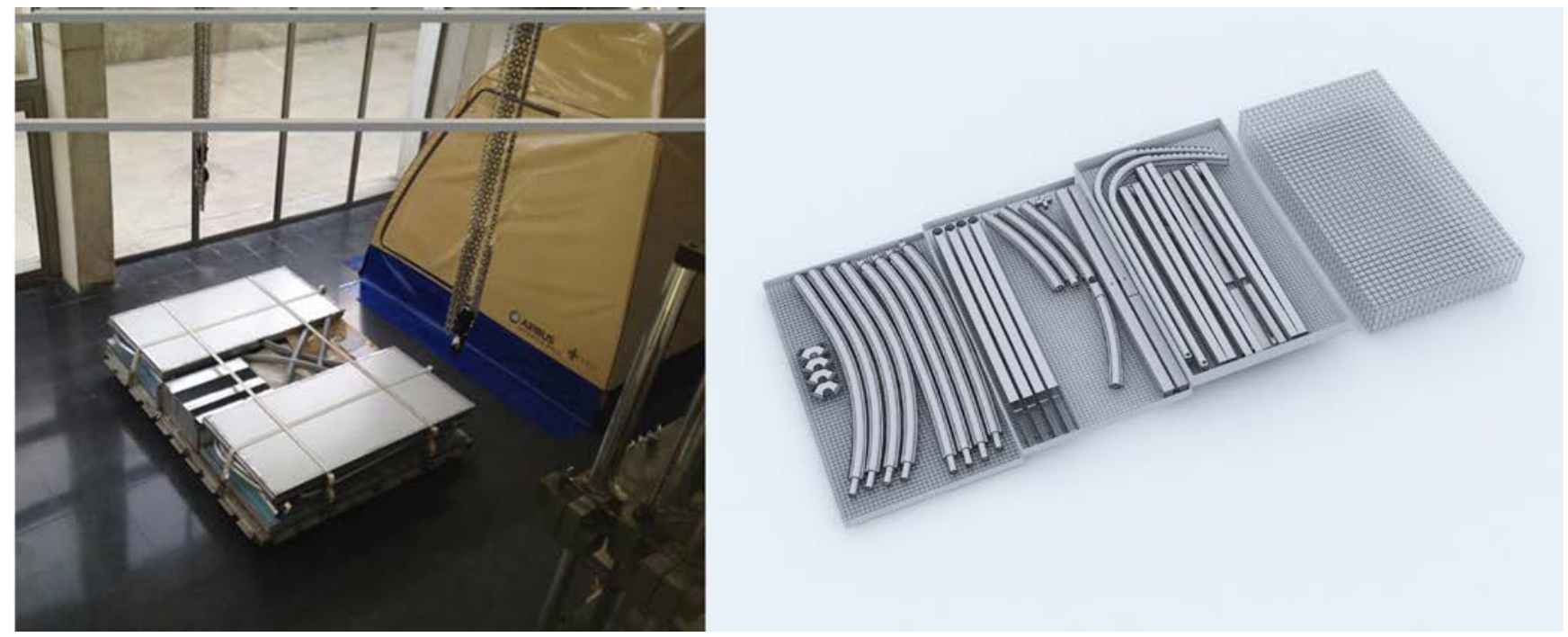

Figura 4. Solución de paquetizado y embalaje de los componentes del sistema. 
C 295 de transporte táctico medio (20) contemplando los datos de combustible, carga útil, etc. Se estima una distancia media de viaje de $7.211,4 \mathrm{~km}$ y una carga por unidad de distancia media transportada de $1.636,98 \mathrm{t}^{*} \mathrm{~km}$. No se van a tener en cuenta los gastos de energía y transporte debidos al movimiento y almacenamiento de la carga en el origen y destino de la misma.

Tanto los impactos derivados del uso propio del producto, totalmente dependientes del modo de utilización del espacio (residuos generados, consumo de agua, etc.) y especialmente los debidos al comportamiento térmico de la misma (su balance térmico), así como del mantenimiento, las reparaciones, las intervenciones necesarias en el producto y la reutilización interna de materiales deberían ser contemplados en su evaluación final. No obstante, la fase de uso no se ha considerado debido a que este tipo de viviendas no tienen un programa condicionado, por ejemplo, sanitario, tampoco disponen de ningún tipo de servicios de apoyo, ni acometida de agua ni de electricidad, por lo que se instalarían letrinas y duchas comunitarias cumpliendo así las recomendaciones internacionales de los agentes humanitarios de viviendas autónomas. De cualquier modo, la parte derivada del uso pertenecería a las dotaciones y servicios comunes de conexión en el Campo de Refugiados, nunca a una prestación individual de cada unidad arquitectónica.

La mayoría de análisis demuestran que sólo entre un número de 10 a 30 de los procesos involucrados son responsables de más del $95 \%$ del impacto ambiental (21). Por otro lado, la inclusión exhaustiva de todos los procesos y su análisis harían que el estudio de ACV deviniera inabarcable. Por eso se realizan diferentes simplificaciones. Quedan excluidos de los límites del sistema:

- Construcción de las infraestructuras (fábricas e instalaciones) de producción de las materias primas, de fabricación de las partes de la VEM y de ensamblaje.

- Fabricación de la maquinaria y equipos auxiliares implicados.

- No se disponen de datos de consumos energéticos debidos a la fabricación de las envolventes, del suelo y del faldón perimetral y los procesos de taladrado, soldadura, galvanizado, cromado y lacado de la estructura principal en las fases de fabricación y montaje.

- No se disponen de datos de tiempo y consumos energéticos empleados en los procesos de verificación visual e inspección por ultrasonidos ni de la verificación de calidad de los diferentes productos.

- La fase de fin de vida del producto está muy ligada al comportamiento del consumidor. La falta de datos concretos, el periodo de vida útil asignado a la Unidad Prototipo (vivienda VEM) y la incertidumbre en los hábitos de reciclado hacen complicado configurar un escenario plausible. En este estudio sólo se contempla la posibilidad de reutilización e incineración de los materiales involucrados en la Unidad Prototipo.

- No se ha considerado el lastre añadido a la vivienda para anclarla en su posición.

\section{ANÁLISIS}

Se ha realizado un análisis comparativo entre todas las partes de la Unidad Prototipo estudiando la carga ecológica en las 18 categorías de impacto intermedias (midpoints) y las tres finales (endpoints). Se indican las diferentes contribuciones de las categorías intermedias a las categorías finales y se consideran los materiales, energía y transporte empleados, así como las diferentes fases por las que pasa el producto. Se ha utilizado el programa SIMAPRO v8.02 (versión 7), herramienta informática desarrollada por PRé Consultants para estudios de ACV que analiza y compara de forma sistemática y consistente los aspectos ambientales de un producto según la norma ISO 14040. Se ha empleado la metodología ReCiPe (22) Midpoint (H), la base de datos de Ecoinvent v3.o (23) (al ser la base de datos de referencia en Europa por su transparencia e independencia) y se ha elegido la perspectiva $\mathrm{H}=$ jerárquica por ser un modelo de consenso, basado en los principios más comunes respecto a plazo temporal y otros.

Existen multitud de categorías de impacto ambiental en un $\mathrm{ACV}$, seleccionando las apropiadas en función del objetivo del estudio, público objetivo y nivel de exactitud de los resultados requeridos. Las categorías de impacto ambiental consideradas son las contempladas por la SETAC (24) (fundada en 1979, con el objetivo de desarrollar la metodología y los criterios del ACV, temas que actualmente lidera) y en la metodología Recipe de Pré Consultants utilizada en este análisis. Con ello se han mejorado los modelos para el cambio climático, la destrucción de la capa de ozono, acidificación, eutrofización, uso del suelo y agotamiento de recursos naturales. A su vez se han actualizado factores de caracterización para algunas categorías de impacto y para el paso de normalización.

También se ha realizado un análisis de impacto ambiental comparativo incluyendo todas las partes que componen la Unidad Prototipo.

\subsection{Resultados y discusión}

Este estudio se ha centrado en la fase de fabricación de todas las partes que constituyen la UPVE, comparándolas todas juntas para tener una visión general de los impactos ambientales, y de la incidencia de la fase final o de gestión de residuos en el impacto ambiental. Se han aplicado procesos de normalización, dividiendo por los siguientes factores de daño: $1,55 \cdot 10^{2}$ (salud humana), 5,13 $\cdot 10^{3}$ (calidad del ecosistema) y $5,94 \cdot 10^{3}$ (recursos); y de ponderación, multiplicando por los factores de daño: 300 (salud humana), 500 (calidad del ecosistema) y 200 (recursos); comparando así las diferentes categorías de impacto bajo cierta perspectiva de igualdad.

En la Figura 5 se pueden analizar las conclusiones acerca del ciclo de vida de los diferentes materiales [los datos están dados en «punto del ecoíndice» $(\mathrm{Pt})$ o ecopunto, que representa una milésima parte de la carga ambiental anual de un ciudadano europeo medio], observando una mayor carga de las partes correspondientes al suelo/base y al soporte inferior, que contribuyen mayoritariamente a la carga en las categorías finales de los daños a la salud humana y a los recursos naturales, y, en menor medida, de la cubierta superior y envolvente exterior. Las partes de la envolvente interior y del faldón perimetral apenas presentan contribución a la carga. La carga ocasionada a los daños al ecosistema es casi nula para todas las partes de la Unidad Prototipo.

En la Tabla 3 se recogen los factores de mayor influencia asociados a las cargas del suelo y del soporte inferior. 

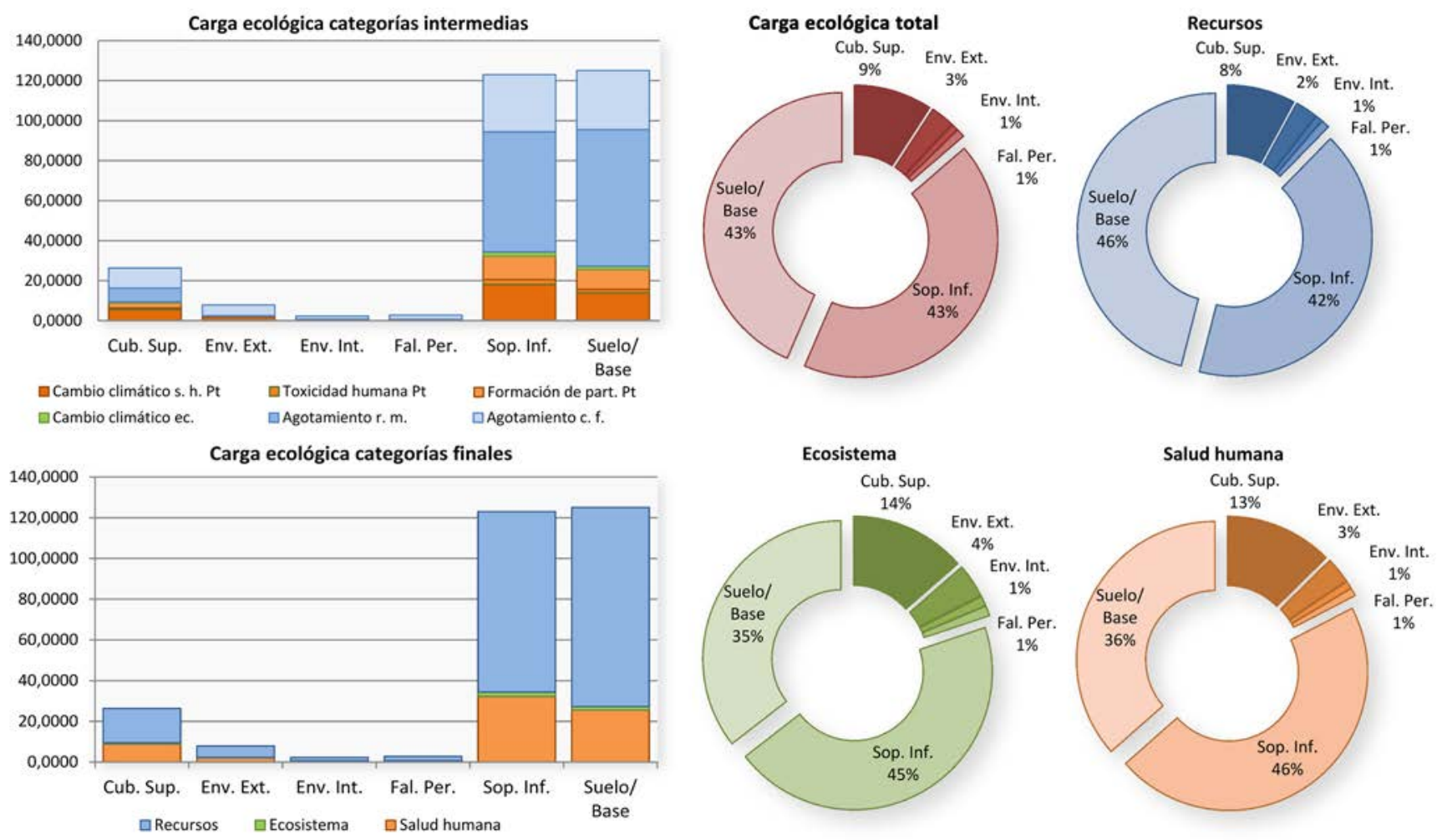

Figura 5. Gráficos de barras de puntuación única (Pt) de la contribución de los diferentes indicadores intermedios y categorías finales a los procesos del conjunto de partes de la Unidad Prototipo. Gráficos porcentuales de la contribución a la carga ecológica en las principales categorías de impacto intermedias y finales asociadas al conjunto de las partes de la Unidad Prototipo.

Tabla 3. Cuadro resumen de cargas asociadas a las diferentes categorías de impacto con indicación de la contribución y los factores de daño para el suelo/base y el soporte inferior.

\begin{tabular}{|c|c|c|c|c|}
\hline \multirow[b]{2}{*}{$\begin{array}{l}\text { Categorías } \\
\text { intermedias }\end{array}$} & \multicolumn{2}{|r|}{ SUELO/BASE } & \multicolumn{2}{|c|}{ SOPORTE INFERIOR } \\
\hline & Contribución & Factores de daño & Contribución & Factores de daño \\
\hline $\begin{array}{l}\text { Agotamiento } \\
\text { combustibles } \\
\text { fósiles }\end{array}$ & $24 \%$ & $\begin{array}{l}\text { Producción de poliol y disocianato de } \\
\text { difenilmetano, extracción de carbón } \\
\text { y producción de gas y petróleo }\end{array}$ & $23 \%$ & $\begin{array}{l}\text { Transporte (camión), operaciones } \\
\text { de extracción del carbón y } \\
\text { producción de petróleo y gas }\end{array}$ \\
\hline $\begin{array}{l}\text { Agotamiento } \\
\text { recursos minerales }\end{array}$ & $55 \%$ & $\begin{array}{l}\text { Producción de material ferroníquel, } \\
\text { Fe y Mn }\end{array}$ & $49 \%$ & $\begin{array}{l}\text { Extracción de materiales: Mo, Fe, } \\
\text { ferroníquel y cromita }\end{array}$ \\
\hline $\begin{array}{l}\text { Cambio climático } \\
\text { (salud humana) }\end{array}$ & $11 \%$ & $\begin{array}{l}\text { Producción de poliol y disocianato } \\
\text { de difenilmetano y producción } \\
\text { de electricidad de alto voltaje } \\
\text { procedente del carbón }\end{array}$ & $15 \%$ & $\mathrm{CO}_{2}, \mathrm{CO}, \mathrm{NOx}, \mathrm{SO}_{2}$ \\
\hline $\begin{array}{l}\text { Formación de } \\
\text { partículas }\end{array}$ & $8 \%$ & $\begin{array}{l}\text { Producción de material } \\
\text { ferrocrómico, y ferroníquel, } \\
\text { producción de calor por gas natural }\end{array}$ & $9 \%$ & $\begin{array}{l}\text { NOx, } \mathrm{SO} 2 \text {, procesos en minas para } \\
\text { extracción de Fe, ferrocromo y } \\
\text { coque }\end{array}$ \\
\hline Toxicidad humana & $1 \%$ & $\begin{array}{l}\text { Producción de material ferroníquel, } \\
\text { producción del acero y producción } \\
\text { del cobre primario }\end{array}$ & $2 \%$ & $\begin{array}{l}\text { Acero de baja aleación, } \mathrm{Zn} \text {, } \\
\text { material de residuo de la escoria de } \\
\text { la fundición, ferroníquel y } \mathrm{Cu}\end{array}$ \\
\hline $\begin{array}{l}\text { Cambio climático } \\
\text { (ecosistema) }\end{array}$ & $1 \%$ & $\begin{array}{l}\text { Producción de poliol y disocianato, } \\
\text { producción de calor por gas natural } \\
\text { y producción de electricidad de alto } \\
\text { voltaje procedente del carbón }\end{array}$ & $2 \%$ & $\begin{array}{l}\text { Transporte (camión), material de } \\
\text { fundición, extracción de carbón } \\
\text { procedente de la mina }\end{array}$ \\
\hline $\begin{array}{l}\text { Categorías } \\
\text { finales }\end{array}$ & Contribución & Factores de daño & Contribución & Factores de daño \\
\hline Salud humana & $21 \%$ & $\begin{array}{l}\text { Producción del material } \\
\text { ferrocrómico, producción de calor } \\
\text { por gas natural y producción de } \\
\text { poliol y disocianato de difenilmetano }\end{array}$ & $26 \%$ & $\begin{array}{l}\text { Transporte (camión), fundición, } \\
\text { mineral de hierro y acero de baja } \\
\text { aleación }\end{array}$ \\
\hline Ecosistema & $1 \%$ & $\begin{array}{l}\text { Producción del material } \\
\text { ferrocrómico, producción de calor } \\
\text { por gas natural y producción de } \\
\text { poliol y disocianato de difenilmetano }\end{array}$ & $2 \%$ & $\begin{array}{l}\text { Transporte (camión), fundición } \\
\text { y operaciones de extracción de } \\
\text { carbón en bruto }\end{array}$ \\
\hline Recursos & $78 \%$ & $\begin{array}{l}\text { Producción del material ferroníquel, } \\
\text { cromita concentrada y producción de } \\
\text { poliol y disocianato }\end{array}$ & $72 \%$ & $\begin{array}{l}\text { Mn, Fe, ferroníquel, cromita, } \\
\text { transporte (camión) }\end{array}$ \\
\hline
\end{tabular}




\subsection{Disposición final. Gestión de residuos}

La fase de fin de vida comienza una vez que el producto, en este caso la Vivienda de Emergencia, ha servido para realizar su función inicial. Se ha valorado la posibilidad de reciclar (a través del mismo sistema de producto -ciclo cerrado de reciclaje- o en un nuevo sistema de producto -ciclo abierto de reciclaje-) o de reutilizar para reducir el impacto ambiental evitando generar residuos.

En este trabajo, como no puede ser de otra manera, primarán como preferencias la «Reducción en origen» y el «Reciclaje y Reutilización», como una forma de disminuir el consumo de materias primas, el consumo energético, el flujo de residuos y las emisiones atmosféricas.

Se analizan como residuos finales de la Vivienda de Emergencia: la estructura principal fabricada en acero al carbono y aleación $\mathrm{Al} 6005 \mathrm{~A}$ (con sus tratamientos), la base/piso fabricada principalmente de poliuretano, las envolventes de poliéster y el faldón perimetral de geotextil (polipropileno + poliéster). Además, se añaden los materiales utilizados para su embalaje y transporte (el plástico y la madera).

Considerando la situación de las personas afectadas se entenderá que, una vez que la Vivienda cumple su objetivo, los materiales que la constituyen tenderán a ser abandonados o reutilizados. Se va a suponer que todos los componentes de la Unidad Prototipo van a ser recogidos y llevados preferentemente a procesos de reciclado (política de impacto ambiental cero) sin descartarse la posibilidad de que algunos materiales puedan reutilizarse e incluso quemarse (parte del palet de madera). No se considerarán en este estudio el gasto energético y de transporte de los materiales a los puntos de reciclaje, de reutilización, a vertederos o a su incineración.

Escenario final para estos materiales:

- Acero S275JR: 90 \% reciclado y $10 \%$ reutilizado.

- Aluminio 6005 A: $100 \%$ reciclado.

- Poliéster (PET): 75 \% reciclado y $25 \%$ al vertedero.

- Polipropileno (PP): 75 \% reciclado y $25 \%$ al vertedero.

- Poliuretano (PUR): 75 \% reciclado y $25 \%$ al vertedero.

- Policloruro de vinilo (PVC): $75 \%$ reciclado y $25 \%$ al vertedero.

- Polietileno baja densidad: $100 \%$ al vertedero.

- Madera: 90 \% reutilizado y $10 \%$ incinerado.

En la literatura existente se han acordado cuantías sobre la energía necesaria utilizada para reciclar determinados materiales. Estos datos aparecen recogidos en el informe Mies (25) y expuestos en la Tabla 4, junto con los porcentajes de los materiales reciclados que componen la Unidad Prototipo. Se puede ver que para la mayoría de los materiales, a excepción de la madera (60 \%), el porcentaje de energía necesaria utilizada en el reciclaje es del orden del $75 \%$ respecto a la energía utilizada para la fabricación del material (acero, plásticos y aluminio).

Se puede destacar que la introducción del proceso de reciclaje (Tabla 5 y Figura 6) para los materiales termoplásticos contribuye a reducir la carga medioambiental aproximadamente

Tabla 4. Energía necesaria para reciclar y fabricar la Unidad Prototipo.

\begin{tabular}{|c|c|c|c|c|c|c|c|}
\hline & \multicolumn{5}{|c|}{ Reciclaje } & \multirow{2}{*}{$\begin{array}{c}\text { Fabricación } \\
\text { Unidad } \\
\text { Prototipo }\end{array}$} & \multirow{2}{*}{$\begin{array}{c}\text { Reciclaje/ } \\
\text { Fabricación }\end{array}$} \\
\hline & & & & $\begin{array}{c}\text { Unidad } \\
\text { Prototipo }\end{array}$ & $\begin{array}{c}\text { Vivienda } \\
\text { (2 unidades) }\end{array}$ & & \\
\hline Material & $\begin{array}{c}\text { Porcentaje } \\
(\%)\end{array}$ & $\begin{array}{c}\text { Energía } \\
(\mathbf{k W h} / \mathbf{k g})\end{array}$ & $\begin{array}{c}\text { Peso } \\
\text { (kg) }\end{array}$ & $\begin{array}{c}\text { Energía } \\
(\mathbf{k W h})\end{array}$ & $\begin{array}{c}\text { Energía } \\
(\mathrm{kWh})\end{array}$ & $\begin{array}{c}\text { Energía } \\
(\mathrm{kWh})\end{array}$ & $\begin{array}{l}\text { Consumo respecto } \\
\text { fabricación (\%) }\end{array}$ \\
\hline Acero & 56,21 & 8,96 & 165,7 & $1.484,64$ & $2.969,27232$ & $1.978,41$ & 75,04 \\
\hline Plásticos & 25,4 & 16,67 & 40,24 & 670,84 & $1.341,68083$ & 892,12 & 75,2 \\
\hline Aluminio & 17,45 & 33,33 & 13,83 & 460,95 & 921,9078 & 614,61 & 75 \\
\hline Madera & 0,95 & 0,5 & 50 & 25 & 50 & 41,5 & 60,24 \\
\hline Total & & & & $2.641,43$ & $5.282,86$ & $3 \cdot 526,64$ & 74,9 \\
\hline
\end{tabular}
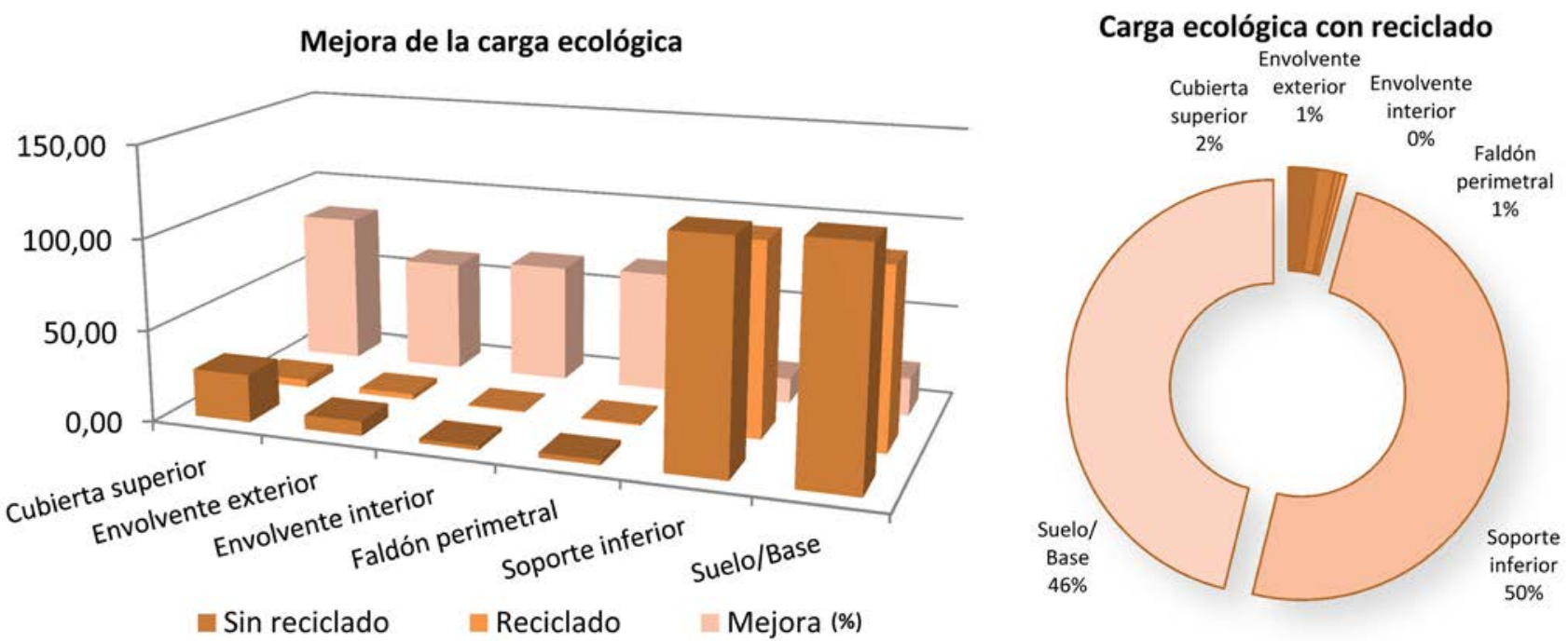

Figura 6. Gráfico de barras de la contribución a la carga ecológica de las diferentes partes de la Unidad Prototipo con indicación de la mejora en la carga ambiental y gráfico porcentual de la contribución a la carga ecológica total considerando procesos de reciclado asociados al conjunto de partes de la Unidad Prototipo. 
Tabla 5. Contribución a la carga ecológica de las diferentes partes de la Unidad Prototipo con indicación de la mejora en la carga ambiental.

\begin{tabular}{|c|c|c|c|c|c|c|c|}
\hline & \multirow[b]{2}{*}{ Unidad } & \multicolumn{6}{|c|}{ Partes/Fases } \\
\hline & & $\begin{array}{l}\text { Cubierta } \\
\text { superior }\end{array}$ & $\begin{array}{c}\text { Envolvente } \\
\text { exterior }\end{array}$ & $\begin{array}{c}\text { Envolvente } \\
\text { interior }\end{array}$ & $\begin{array}{c}\text { Faldón } \\
\text { perimetral }\end{array}$ & $\begin{array}{l}\text { Soporte } \\
\text { inferior }\end{array}$ & Suelo/Base \\
\hline Sin reciclado & $\mathrm{Pt}$ & 26,35 & 7,90 & 2,30 & 2,80 & 123,02 & 125,09 \\
\hline Reciclado & $\mathrm{Pt}$ & 4,41 & 3,05 & 0,80 & 0,94 & 106,15 & 98,95 \\
\hline Mejora & $\%$ & 83,25 & 61,34 & 65,01 & 66,41 & 13,72 & 20,90 \\
\hline
\end{tabular}

entre un 61 y $66 \%$. Para la cubierta superior la reducción del impacto ambiental es muy considerable ( $83 \%$ ), siendo muy inferior para las partes del suelo/base y del soporte inferior. Estos últimos contribuyen con una gran carga ecológica con respecto al resto de partes, posiblemente debido a la influencia de los materiales aleantes.

\section{CONCLUSIONES}

Se han determinado las cargas ambientales asociadas a las diferentes partes de la Unidad Prototipo de la Vivienda de Emergencia (VE) y se han aplicado procesos de disposición final del producto: reciclado, reutilización, incineración, etc.

Sólo las siguientes categorías intermedias, analizadas mediante la metodología Recipe, contribuyen de manera importante a la carga ambiental según procesos de ponderación (medidas en ecopuntos): cambio climático en la salud humana, toxicidad humana, formación de partículas, cambio climático en el ecosistema, agotamiento del recurso mineral y agotamiento del combustible fósil.

Se puede concluir que la mayor carga ambiental es aportada generalmente en la categoría intermedia del agotamiento de los combustibles fósiles, excepto para las partes del soporte inferior y del suelo que lo hacen en la categoría del agotamiento de los recursos minerales. Para la formación del material particulado la contribución a la carga se encuentra entre un 5 y $10 \%$, para la categoría del cambio climático en el ecosistema entre un 11 y 21 \% y es mínima para el resto de categorías intermedias, como la toxicidad humana y el cambio climático en el ecosistema, entre el 1 y $2 \%$.

Con respecto a las categorías finales se destaca una mayor aportación a la carga en la categoría de los recursos (64-78 $\%)$, sensiblemente menor en la categoría de impacto de la salud humana (21-33 \%) y mínima para la categoría de los daños ocasionados a la calidad del ecosistema (1-3 \%).

En relación al suelo/base de la Unidad Prototipo se debe de evitar el empleo del PVC (policloruro de vinilo) por sus perjuicios para la salud y el medio ambiente. El PVC contamina en todo su ciclo de vida (durante su producción, su utilización y como residuo), además de liberar ácido clorhídrico y otros gases tóxicos en caso de incendio. El poliuretano (PUR) tiene un alto impacto en el efecto invernadero, la contaminación atmosférica y la reducción de la capa de ozono y el poliestireno (PS) sobre la acidificación, la contaminación atmosférica, los metales pesados y la energía. Ambos materiales son componentes del panel tipo sándwich del suelo.

El soporte inferior, fabricado en acero al carbono-manganeso, tiene un alto impacto ambiental por aleantes como el Mn, P, Si, S y Al.
En función de lo anterior, parece interesante rediseñar los materiales que componen el suelo y el soporte inferior de este tipo de vivienda de emergencia por su alto índice de impacto respecto a las otras partes de la misma. Se pretende promover productos de las mismas categorías que puedan reducir los efectos ambientales adversos, contribuyendo así a un uso eficaz de los recursos y a un elevado nivel de protección del medio ambiente.

Las fases estudiadas de distribución y embalaje del producto presentan daños de contribución a la carga de un $4 \%$ y de un $1 \%$, respectivamente. La fase de uso y mantenimiento debería ofrecer valores importantes de contribución a la carga, que no son contemplados en la fase estudiada, a la espera de conocer las condiciones finales particulares de su emplazamiento y de su puesta en servicio.

Este tipo de construcciones de Viviendas de Emergencia debe intentar llevarse al impacto nulo, considerándose como experiencia piloto para abordar posibles misiones a otros países y promover la existencia de sinergias entre las diferentes iniciativas de proyectos afines a éste. De esta forma se fomentará el desarrollo de nuevos productos, la mejora de metodologías de diseño y la creación de un Centro de Control de producción limpia como institución referente que primara el impulso a la transformación productiva. Se pretende de esta forma dar un paso importante hacia una sociedad a corto plazo de cero-residuos y cero-contaminantes, creando las bases técnicas para una futura implementación.

Con la introducción de los procesos de disposición final de los materiales analizados, se deduce que todos ellos son beneficiosos para el medio ambiente. Se reduce mucho la carga en las partes de la envolvente interior (89 \%) y la cubierta superior (87\%), para el faldón perimetral (66 \%) y para la envolvente exterior (61\%), se producen valores intermedios y para el soporte inferior (13\%) y el suelo (20 \%), se obtienen valores muy bajos debido al uso de aceros aleados.

Teniendo en cuenta estos beneficios para el medio ambiente por la utilización de procesos de disposición final, se concluye que la contribución a la carga final es la siguiente: cubierta superior (2\%), envolvente exterior (1\%), envolvente interior (casi nulo), faldón perimetral (1\%), soporte inferior (50 \%) y suelo/base (46\%).

Estos resultados apoyan el objetivo de implementar el Cradle to Cradle como paradigma de desarrollo socio-económico de diseño, de modo que se consideren todos los materiales involucrados en el proceso como «nutrientes». Es necesario optimizarlos respecto a la salud humana y el medio ambiente permitiendo la inversión necesaria en la producción y distribución del producto en el mercado. Se crearía así un incentivo de recuperación de los mismos en las diferentes fases de uso a través de procesos de reciclado, reutilización, etc., propiciando el diseño de sistemas de «logística inversa» cuyo 
objetivo sea la recuperación de nutrientes en los materiales de una manera efectiva y eficiente.

Se considera necesaria la búsqueda de la Etiqueta Ecológica Europea del producto como una parte importante de la política comunitaria para mejorar la actuación ambiental. La meta será poder obtener en un determinado plazo de tiempo una economía circular y un crecimiento verde: producción limpia, huella de carbono, energías renovables, cero residuos, valorización de los materiales, productividad, calidad, etc.

\section{AGRADECIMIENTOS}

Los autores quieren agradecer a Airbus Defense \& Space por la financiación recibida a través de su programa de compensación industrial offset para el desarrollo del Proyecto de Viviendas de Emergencia Militar (VEM), al Vicerrectorado de Investigación de la Universidad CEU-San Pablo y a la Escuela Politécnica Superior de la Universidad CEU-San Pablo por su apoyo.

\section{REFERENCIAS}

(1) Ros García, J. M. (coord.) (2015). Arquitecturas de emergencia. Cuadernos de investigación, n. ${ }^{0}$ 05, p. 169. Madrid: CEU Ediciones.

(2) Ros García, J. M., González, R., Hormigos, S. (2014). Evaluación de la influencia del factor de forma de una estructura de nervios cuatripartitos bajo solicitaciones cuasiestáticas. Pensamiento Matemático, IV(2): 75-89.

(3) Clementes, R. (2000). Guía Completa de las Normas ISO 1400o, p. 104. Barcelona: Ediciones Gestión.

(4) AENOR-CEN (2006). UNE-EN ISO14040 Gestión ambiental. Análisis de ciclo de vida. Principios y marco de referencia. AENOR.

(5) AENOR-CEN (1999). UNE-EN ISO14041 Gestión ambiental. Análisis de ciclo de vida. Definición del objetivo y alcance y el análisis de inventario. AENOR.

(6) AENOR-CEN (2001). UNE-EN ISO14O42 Gestión ambiental. Análisis de ciclo de vida. Evaluación de impacto del ciclo de vida. AENOR.

(7) AENOR-CEN (2001). UNE-EN ISO14043 Gestión ambiental. Análisis de ciclo de vida. Interpretación del ciclo de vida. AENOR.

(8) AENOR-CEN (2006). UNE-EN ISO14044 Gestión ambiental. Análisis de ciclo de vida. Requisitos y directrices. AENOR.

(9) AENOR-CEN (2012). UNE-EN ISO15804 Sostenibilidad en la construcción. Declaraciones ambientales de producto. Reglas de categoría de productos básicas para productos de construcción. AENOR.

(10) AENOR-CEN (2012). UNE-EN ISO15978 Sostenibilidad en la construcción. Evaluación del comportamiento ambiental de los edificios. Métodos de cálculo. AENOR.

(11) AENOR-CEN (2012). UNE-EN ISO15643 Sostenibilidad en la construcción. Evaluación de la sostenibilidad de los edificios. Parte 1: Marco general. AENOR.

(12) AENOR-CEN (2010). UNE-EN ISO21930 Sostenibilidad en la construcción de edificios. Declaración ambiental de productos de construcción. AENOR.

(13) AENOR-CEN (1996). UNE-EN ISO15O-O4O Análisis del ciclo de vida. Principios generales. AENOR.

(14) ISO (2012). ISO/TR-14049. Environmental management -- Life cycle assessment -- Illustrative examples on how to apply ISO 14044 to goal and scope definition and inventory analysis. International Organization for Standardization (ISO).

(15) Antón, M. A. (2004). Metodología del análisis del ciclo de vida, en Utilización del Análisis del ciclo de vida en la evaluación del impacto ambiental del cultivo bajo invernadero mediterráneo. Barcelona: Universidad Politécnica de Cataluña.

(16) AENOR-CEN (2007). UNE-EN ISO10025-5 Productos laminados en caliente de aceros para estructuras. Parte 5: Condiciones técnicas de suministro de los aceros estructurales con resistencia mejorada a la corrosión atmosférica. AENOR.

(17) AENOR-CEN (2015). UNE-EN 10o88-1 Aceros inoxidables. Parte 1: Relación de aceros inoxidables. AENOR.

(18) Asociación sin ánimo de lucro eco-union Mediterránea. http://www.eco-union.org/

(19) Estevan, M. T. (1992). El Medio Ambiente en el Acta Única Europea, p. 157-173, Cuadernos de Estrategia. Madrid: Instituto Español de Estudios Estratégicos, Ministerio de Defensa.

(20) EADS-Airbus Military. http://www.airbusgroup.com/int/en.html

(21) Curran, M. A. (1996). Environmental life-cycle assessment. The International Journal of Life Cycle Assessment, 1(3): 179-179, doi: http://dx.doi.org/10.1007/BFo2978949.

(22) ReCiPe methodology for Life Cycle Impact Assessment (LCIA). http://www.lcia-recipe.net/

(23) Ecoinvent Centre. Database http://www.ecoinvent.org/

(24) SETAC (1999). Life Cycle Assessment and Conceptually Related Programmes, p. 28. Brussels: Society for Environmental Toxicology and Chemistry - SETAC (Europe Working Group).

(25) Gestión del impacto ambiental. Estrategia del grupo PRISA. 